\title{
DISCOVERY OF INFRARED STARS IN GLOBULAR CLUSTERS IN THE MAGELLANIC CLOUDS AND THEIR LIGHT VARIATIONS
}

\author{
S. NISHIDA, T. TANABÉ, S. MATSUMOTO, T. ONAKA AND Y. NAKADA \\ University of Tokyo, Japan \\ snishida@mtk.ioa.s.u-tokyo.ac.jp, ttanabe@mtk.ioa.s.u-tokyo.ac.jp \\ K. SEKIGUCHI \\ National Astronomical Observatory of Japan, Japan \\ AND \\ I. S.GLASS \\ South African Astronamical Observatory, South Africa
}

A systematic near-infrared survey was made for globular clusters in the Magellanic Clouds. Two infrared stars were discovered in NGC419 (SMC) and NGC1783 (LMC). NGC419 and NGC1783 are well-studied rich globular clusters whose turn-off masses and ages are estimated $M_{\text {To }} \sim 2.0 M_{\odot}$ and $\tau \sim 1.2 \mathrm{Gyr}$ for NGC419, and $M_{\mathrm{TO}} \sim 2.0 M_{\odot}$ and $\tau \sim 0.9 \mathrm{Gyr}$ for NGC1783, respectively.

The periods of the infrared light variations were determined to be $540 \mathrm{~d}$ for NGC419IR1 and to be $480 \mathrm{~d}$ for NGC1783IR1, respectively. Comparison of the measurements with the period $-K$ magnitude relation for carbon Miras in the LMC by Groenewegen and Whitelock(1996) revealed that the $K$ magnitudes of the infrared stars were fainter by about $0.3-0.8$ magnitude than those predicted by the $P-K$ relation. This deviation can be explained if the infrared stars are surrounded by thick dust shells and are obscured even in the $K$ band. The positions of NGC419IR 1 and NGC1783IR1 on the $P-K$ diagram suggest that AGB stars with the main sequence masses of about $2 M_{\odot}$ start their heavy mass-loss when $P \sim 500 \mathrm{~d}$. 